\title{
Estructura y rasgos discursivos característicos de narraciones espontáneas en lengua de señas chilena: su valor para una educación bilingüe*
}

\author{
Fabiola Otárola $\mathrm{C} .{ }^{* *}$, \\ Nina Crespo A.**
}

RESUMEN

Es necesario dar cuenta de diversos aspectos de la lengua de señas chilena (LSCh) para su mejor aprovechamiento en la educación bilingüe de los sordos. Por este motivo, el objetivo de este trabajo es describir tres narraciones producidas por signantes de LSCh. Los resultados permiten señalar que estas poseen una estructura característica en la cual cumplen roles centrales la elaboración de escenarios imaginarios, la representación de acciones y el uso de clasificadores. A partir de allí, se señalan algunas propuestas educativas que aprovechen estos rasgos de la LSCh.

Palabras clave: narración, lengua de señas, espacio, clasificadores.

Structure and distinctive discursive features of spontaneous stories in Chilean Sign Language: Their value for a Bilingual Education \begin{abstract}
It is necessary to account for various aspects of Chilean Sign Language (LCSh) for its best use in bilingual education of deaf people. Therefore, the aim of this paper is accounting for three narratives produced by signers of LCSh. The results would note that these have a characteristic structure in which the development of imaginary scenarios, the action representation and the use of classifiers play a central role. From there, it points to some educational proposals that take advantage of these traits of LCSh.
\end{abstract}

Key words: narrative, sign language, space, classifiers.

* Este trabajo forma parte del Proyecto FONDECYT II30420.

* Fabiola Otárola es Profesora Diferencial con Mención en Trastornos de Audición y Lenguaje de la Universidad Metropolitana de Ciencias de la Educación (UMCE); Candidata a Doctor en Lingǘstica de la Pontificia Universidad Católica de Valparaíso (PUCV), Chile. Investigadora del equipo FONDECYT II30420 20I3- 2015 "Factores lingüísticos y cognitivos que se relacionan con el desarrollo de la complejidad sintáctica oral en los primeros años escolares" de la Dra. Nina Crespo. Pontificia Universidad Católica de Valparaíso. E-mail: fabiolaotarolac@gmail.com

Nina Crespo es Doctora en Lingüística y Profesora de la Pontificia Universidad Católica de Valparaíso (PUCV), Chile. Investigadora responsable del proyecto FONDECYT II30420 2013- 2015 "Factores lingüísticos y cognitivos que se relacionan con el desarrollo de la complejidad sintáctica oral en los primeros años escolares". Pontificia Universidad Católica de Valparaíso. E-mail: ncrespo@ucv.cl 


\section{Introducción}

La lengua de señas (LS) es un lenguaje viso-gestual, creado y utilizado por las personas sordas de todas las edades, de diferentes comunidades y culturas (Valli \& Lucas, 2000; Minami, 2002). Con variaciones entre las diferentes LS, lo que las hace constituirse como lenguas particulares, con rasgos distintivos, pero con un mismo principio estructural: multidimensional, viso-gestual, autónoma de las lenguas orales con una estructura lingüística determinada.

La persona sorda utiliza para la construcción de su discurso varias herramientas que le proporciona su lengua, dependiendo del tipo de información que transmite y del propósito del mensaje. De esta manera, puede utilizar, tanto simultánea como alternadamente, dactilología (alfabeto manual), clasificadores, gestos no manuales, movimientos corporales y uso del espacio (Crespo, 1993).

A raíz de las particularidades de esta lengua, los métodos de investigación de la LS deben reconocer las configuraciones manuales, el uso del espacio, los gestos y los movimientos corporales. Es, además, necesario tener en cuenta que las lenguas de señas, por un lado, carecen de versión escrita y, por otro, son totalmente dependientes de las lenguas habladas. Por este motivo, deben ser abordadas a través de metodologías que den cuenta de sus características y que consignen los diferentes niveles que constituyen sus mensajes. Por lo tanto, el desarrollo de sistemas de notación video-grabada ha sido para las lenguas de señas un aspecto fundamental en la investigación lingüística.

En Chile las investigaciones sobre la Lengua de Señas Chilena (LSCh) comenzaron desde los años noventa, por lo cual es un área de investigación en desarrollo. Las investigaciones realizadas han apuntado a la descripción de aspectos gramaticales específicos, como la estructura subléxica (Adamo, I993), la pronominalización (Cabrera, I993), la estructuración sintáctica (Pilleux, I992; Adamo, 1993; Adamo, Cabrera, Lattapiat \& Acuña, 1999) y los fenómenos semánticos que ella presenta (Ibáñez, Becerra, López, Sirlopú \& Cornejo, 2005; Becerra, 2008). Asimismo -tal como en otros países- se comienzas a abordar temáticas que revalorizan esta 
lengua como sistema de comunicación natural de las personas sordas (Adamo, Acuña, Cabrera \& Cárdenas, 1997; Adamo, Cabrera \& Acuña, 2005). Además, se publica el primer diccionario de LSCh (Pilleux, I992), se realizan estudios que señalan las implicancias de la Educación Bilingüe en la educación del sordo (Lissi, Svartholm \& Gonzales, 2012) y se enfatiza en las dificultades que los estudiantes sordos manifiestan en aprendizajes escolares, específicamente, en la lectura y la escritura (Miranda, I997; Herrera, 2003, 2007 y 2009). Finalmente, Acuña, Adamo, Cabrera y Lissi (2012) realizaron la primera investigación de carácter exploratorio y descriptivo de las narraciones de niños sordos en tareas de recontado, proponiendo así uno de los pocos enfoques discursivos del LSCh.

En respuesta a lo anterior, este estudio intenta contribuir al conocimiento de cómo se construye el discurso en LSCh, considerando la narración y, más específicamente, el relato de experiencias personales. A través de esta, se busca el reconocimiento de la estructura y los rasgos discursivos característicos presentes en las narraciones en LSCh. Esto se lleva a cabo por medio de una metodología de estudio que apunta a entender la lengua en sí misma, sin referirla a paradigmas o elementos de las lenguas orales de los cuales difieren considerablemente. Solo a partir de allí, es posible hacer propuestas educativas que consideren de manera más clara, quién es la persona sorda y qué se puede hacer con su lenguaje en la educación.

El presente trabajo se configura como una propuesta entre dos disciplinas: la Lingüística y la Educación; en otras palabras, interesa aquí indagar ciertos rasgos de las narraciones construidas en LSCh para reflexionar acerca de su valor en la propuesta de Educación Bilingüe. Por este motivo, en el marco teórico se ahonda en dos elementos: la valoración de una propuesta bilingüe de educación para las personas sordas, por un lado, y por otro, la caracterización de la modalidad narrativa y de su materialización en las LS.

A continuación, se presenta tanto la metodología como el trabajo de campo que aborda las narraciones en LSCh y se da cuenta de los principales resultados. Finalmente, en la conclusión, se considera el potencial valor de estos hallazgos para enriquecer la propuesta de Educación Bilingüe. 


\section{La educación bilingüe de los niños sordos}

La educación y el desarrollo de la LS han estado estrechamente ligados desde sus inicios por un aspecto fundamental que responde a las preguntas: ¿cómo adquiere la lengua una persona sorda?; ¿la forma de adquisición será distinta a la manera cómo la adquiere un oyente? Pues sí, los niños oyentes aprenden su lengua con naturalidad en todos los espacios posibles en los cuales interactúan. Esto ocurre principalmente al interior de sus familias, ya que están dotados para percibir los rasgos de la lengua oral y conectarla con el contexto inmediato. Dicho fenómeno no se da en el caso de un niño sordo, la lengua oral no es directamente percibida por él, sino que desde las propuestas oralistas se le enseña en una forma descontextualizada en la cual prima lo formal frente a lo funcional. Muchos investigadores han llamado la atención acerca del posible daño que esto conlleva a los niños sordos, perjuicios que se ven representados en un desarrollo cognitivo y comunicativo más disminuido (Crespo, 1993).

La única excepción sería el caso de los niños sordos, hijos de padres sordos, que poseen un desarrollo lingüístico similar al de los oyentes, pues perciben la lengua visogestual de sus padres (Pérez, 2006). Sin embargo, ellos representan el 6\% de la población total; el otro 94\% de la población tiene padres oyentes (Herrera, 2010). Por tanto, para este gran porcentaje de la población sorda, la única oportunidad de apropiarse de una lengua que puedan percibir directamente y conectarla con el contexto, se da en las escuelas bilingües donde se usan las señas; ellas constituyen ambientes lingüísticos apropiados para adquirir la lengua, la cultura y desarrollarse social y emocionalmente (Skliar, 1998; Lissi et al., 2012).

Sin embargo, esta modalidad educativa solo es posible desde hace muy pocos años. Hasta finales de los años setenta, el lenguaje hablado, en la mayoría de los países era la única modalidad de educación aceptada en las aulas para niños sordos. Esta situación se basaba en el prejuicio de que la LS no permitía a los estudiantes acceder al desarrollo cognitivo e impedía que comunicaran ideas abstractas, ya que aún se creía que la LS solamente podía transmitir ideas 
concretas. No obstante, como ya se señaló, el oralismo en sí mismo no fue muy exitoso, muchos niños sordos aprendieron a hablar y a leer los labios, pero en el proceso, su desarrollo psicolingüístico se vio afectado (Lissi et al., 2012).

Posteriormente, se implementó en la educación de los niños sordos el lenguaje hablado signado, en el cual se utilizaban señas y gestos simultáneamente con el habla (idioma del país) y cualquier otro medio visual para la transmisión de la información. Esta línea educativa tampoco funcionó como se esperaba, ya que los estudiantes no lograban avanzar de la enseñanza secundaria dado que seguían evidenciando una detención en su desarrollo psicolingüístico y, como consecuencia del mismo, no lograban avanzar en la educación secundaria. Finalmente, se desarrollaron estudios sobre las LS naturales que los sordos utilizaban al interior de sus comunidades en diferentes países (Liddell \& Metzger, 1998). Esto permitió observar que las LS se han desarrollado, al igual que las lenguas habladas, como sistemas lingüísticos muy complejos y, por esto, cada seña no es necesariamente equivalente a una palabra en una lengua hablada, pues una sola seña incluye gran cantidad de información (Schembrie, 2003). En otras palabras, se descubrió que las LS eran independientes de las lenguas orales y, además, que eran distintas entre sí. De esta manera, por ejemplo, el lenguaje de señas de las comunidades sordas de Argentina es diferente de la utilizada en las comunidades sordas chilenas.

A partir de allí, surge el reconocimiento por la LS y la importancia que esta tiene en la formación de las personas sordas, creándose una propuesta nueva llamada Educación Bilingüe o Bilingüe-Bicultural para los niños y jóvenes sordos. Estas defienden el uso de la LS en la educación del sordo y el desarrollo paralelo de la lengua correspondiente a la cultura en que el individuo se inserta, principalmente, a través de la exposición a textos escritos (Svartholm, 1984).

En Chile este proceso educativo ha sido tardío, ya que el fuerte arraigo del oralismo y la comunicación total (oralidad signada), aún presentes en las escuelas, ha puesto mucha resistencia a los cambios (Adamo, Cabrera \& Acuña, 2008). Solo en el año 2000 se da inicio a la primera escuela con un proyecto educativo bilingüe-bicultural 
(Herrera, 2010; Lissi et al., 2012), el cual en los últimos años ha logrado ser transmitido a otras escuelas en el país.

Los cambios existentes en la educación especial en Chile, respecto a las nuevas propuestas educativas bilingües, favorecen el desarrollo de la LS en espacios socio/educativos. Es más, la Ley No 20.602 reconoce la LSCh como medio de comunicación natural de la comunidad sorda de nuestro país, dándole un estatus importante para la educación y la formación de las personas sordas. Sin embargo, es necesario conocer con más profundidad la LSCh para mejorar su eficacia como herramienta educativa. Esto ha permitido un incremento en el desarrollo de investigaciones acerca de los rasgos característicos de esta lengua. Se considera que observarlos en el marco de la producción del discurso narrativo permitirá comprender de manera más acabada la potencialidad semiótica de los diversos recursos de este sistema visogestual.

\section{La narración}

Las narraciones pueden definirse como historias sobre eventos reales o ficticios, cuya producción implica la concatenación de unidades que revelan información sobre el contexto situacional, el personaje, las acciones, las motivaciones y emociones que se desencadenan en estos (Petersen, Gillam \& Gillam, 2008).

La narración, al igual de lo que ocurre con las lenguas habladas, se torna una modalidad discursiva interesante para el estudio de la LS, dentro de la cual se pueden observar diferentes procesos lingüísticos que permiten describir a las diversas LS (Metzger, I995; Massone, I996; Liddell \& Metzger, I998; Oviedo, I996; Morgan, 2005; Mulrooney, 2009; Earis \& Cormier, 2013, entre otros). Además, el género narrativo como modalidad autogestionada (Owens, I995) se constituye como un reflejo de la cultura de los hablantes o signantes, incluso más que las conversaciones (Minami, 2002), ya que es un instrumento de transmisión cultural; así lo señalan Stromqvist y Verhoeven (2004:3):

Narratives can be seen as an important device for human communication. People use narratives to recapitulate past experience by matching a verbal sequence of clauses to the sequence of events which actually occurred. Thus, the 
reproduction of narratives can be seen as a major vehicle for cultural transmission.

Cabe señalar que, debido a que es una lengua sin versión escrita, la LS se transmite, fundamentalmente, frente a frente lo que convierte a la narración en una particular modalidad discursiva que debe ser registrada en la interacción, en la cual presenta ciertos rasgos discursivos característicos.

\section{La estructura narrativa de la lengua de señas y sus rasgos discursivos característicos}

Las investigaciones que han considerado la narrativa en LS han trabajado fundamentalmente con el método de recontado (Oviedo, 1996; Morgan, 2005; Mulrooney, 2009; Acuña et al., 2012), en el cual se pide a los entrevistados que refieran un cuento (muchas veces de la tradición de lengua oral) que han visto o han leído con anterioridad. El principal problema de este tipo de trabajos está dado por la influencia del input inicial en la estructura narrativa que producen los signantes. En otras palabras, dadas las particularidades propias de las LS, no es posible asegurar que esa estructura se repetiría en el caso de una narración espontánea.

Aun así, ha sido factible indicar algunos rasgos que parecieran típicos de las narraciones en lengua de señas. De esta manera, autores como Oviedo (1996) y Morgan (2005), usando la tarea de recontado, han descrito que la estructura narrativa en la LS consiste en un primer momento o sección inicial en la descripción de un personaje del relato, por lo general, es quien inicia las acciones. En esta identificación se le da al personaje un nombre (que puede ser propio o común) asociado a un apodo ${ }^{\mathrm{I}}$ y se le asignan cualidades, tales como algún rasgo físico notorio, o se informa si se trata de un niño o un anciano, etc. En esta sección, también, se presenta el contexto en el que se desarrollan las acciones y los otros personajes que participan en los eventos. Además, es aquí donde se hace presente un particular fenómeno

En la cultura sorda la asignación de apodos a las personas se realiza para evitar el deletreo de sus nombres, lo que podría resultar tedioso y entorpecer el ritmo del discurso. Estos apodos son por lo general gestos referidos a algún rasgo físico destacado en cada persona. 
de las narraciones signadas, en el cual se asume ser el protagonista del relato, de modo que las acciones realizadas por el señante son entendidas como las acciones ejecutadas por el personaje principal. Este recurso se denomina en las investigaciones de LS cambio de rol o construcción del personaje (Mulrooney, 2009).

Luego, en un segundo momento, se desarrolla la historia con la construcción secuencial de espacios mentales o escenarios correspondientes a la secuencia narrativa (Oviedo, 1996), en la que participan los distintos personajes. Cuando surge un personaje nuevo en el relato se le asigna una ubicación espacial específica, la cual permitirá al señante y al interlocutor identificarlo a través de una acción deíctica en el espacio establecido (Emmorey, Korpics \& Petronio, 2008) o a través de su apodo. Significativo es el uso de la mirada en la introducción o cambio en los personajes durante el discurso narrativo, lo que se articula junto a elementos gramaticales como verbos o no gramaticales como movimientos corporales (Oviedo, I996; Thomson, Emmorey \& Kluender, 2006). La coherencia que proporcionan la deixis, la mirada, los gestos no manuales y la construcción de rol, entre otros, permiten que los segmentos de la narración se relacionen en forma sucesiva y causal, pero que, además, cada uno de ellos represente una condición que hace que el siguiente sea posible o necesario (Cruz, 2008).

A partir de allí, Cruz (2008) señala que algunos de sus rasgos discursivos característicos en las narraciones en LS son: a) uso icónico y dé́ctico del espacio y b) la preferencia por el uso de clasificadores o proformas.

El uso del espacio señante es usado para hacer referencia a los distintos eventos que se van construyendo, o los roles que el señante desempeña a través de un cambio de orientación de su cuerpo en el espacio para la construcción del rol (Cruz, 2008). De esta forma, las marcas espaciales al interior del discurso narrativo permiten la claridad del señante en las ubicaciones espaciales que se adscriben a cada una de las entidades. Es lo que da cohesión al discurso (Acuña, Adamo \& Cabrera, 2009). Los clasificadores aparecen en el texto narrativo cuando surge una entidad nominal o bien un conjunto de estas. Posteriormente, al 
continuar la historia se hace referencia a la entidad anteriormente nombrada, entregando información sobre su acción y localización a través de configuraciones manuales. Estas construcciones permiten dar cuenta de la relación espacial entre la persona u objeto y, también, permiten hacer uso del espacio físico para situar a través de la señalización las acciones que realizan los personajes sobre o hacia algo (Acuña et al., 2012). Además, dan cuenta de rasgos característicos (por ejemplo, gordo, delgado, largo, redondo) o propiedades humanas o animales de los personajes. Estos rasgos discursivos están relacionados comúnmente a verbos de movimiento, indicando la dirección o tipo de movimiento de la entidad a la cual se refieren.

\section{Metodología}

La metodología utilizada en este estudio exploratorio es cualitativa, dado que el objetivo es describir las narraciones espontáneas de signantes sordos usuarios de la LSCh. Para esto, se analizan cuatro narraciones de experiencias personales producidas por sujetos sordos, extraídas de una entrevista grupal o focus group, definida como una técnica de levantamiento de datos, la cual gira alrededor de una temática propuesta por el investigador. En este caso la temática central propuesta a los narradores fue relatar el momento en que descubrieron que eran sordos, actividad que se realizó en un espacio cercano a los participantes y guiada por una persona conocedora y fluente de la LSCh.

\subsection{Sujetos}

Se incorpora a esta investigación exploratoria a cuatro sujetos sordos: tres hombres y una mujer, todos usuarios de la LSCh. Dos de los participantes trabajan como profesores o co-educadores en una escuela para sordos con enfoque bilingüe ${ }^{2}$. Todos los voluntarios

2 La escuela a la que se hace mención es la Escuela para Sordos "Dr. Jorge Otte Gabler", ubicada en Santiago. En ella se imparte formación para niños y jóvenes sordos dentro de un contexto educativo bilingüe, es decir donde se da énfasis al aprendizaje y uso de LSCh (LI) la cual es transmitida principalmente por sus profesores y co-educadores sordos. Como segunda lengua (L2) se utiliza la lengua escrita, la cual complementa a la LSCh en los procesos regulares de enseñanza. La L2 es impartida preferentemente por profesores oyentes fluentes en LSCh. 
poseen pérdida auditiva profunda o sordera, de carácter congénito y sus edades fluctúan entre los 35 y 50 años. $\mathrm{Al}$ inicio de la dinámica grupal, se les informó de manera general de los alcances de este trabajo, se informa que la conversación será video-grabada y se asegura la confidencialidad. Por su parte, los participantes firman un consentimiento informado, donde autorizan que el investigador utilice los datos de este estudio.

\subsection{Recolección de los datos}

Para elicitar los textos narrativos se considera el modelo propuesto por Labov y Waletsky (1967), quienes plantean que para el estudio de los relatos se deben observar estructuras narrativas simples, las cuales se encuentran principalmente en las versiones orales de experiencias personales y no en la de los expertos. Así, siguiendo a Schembri et al. (2013) respecto a la estrategia de recolección de los textos narrativos, la toma de muestra tuvo el formato de una entrevista semi-estructurada (Mayan, 200I). En el transcurso de la misma, se le solicitó al voluntario que narrara el momento en que se dio cuenta de que era sordo.

Los informantes fueron abordados en parejas que participaron en momentos diferentes. De esta manera, tanto la primera como la segunda pareja aparecieron incorporadas en un grupo mayor de personas sordas que formaban un semicírculo frente a la investigadora. Esta posición permitía que los signantes pudieran observar con claridad la conversación de sus compañeros y así facilitar la interacción entre ellos, dándole un formato de conversación espontánea. La actividad tuvo una duración de I5 minutos aproximadamente, en la cual se registró la participación equitativa de todos los participantes.

\subsection{Criterios para transcripción y presentación de la información signada}

La descripción de las narraciones elicitadas está basada en el Sistema de Glossa en LS o Glossing Sings (Valli \& Lucas, 2000). Este proceso difiere de la traducción, pero mantiene ciertos rasgos 
de la lengua. La glossa será una palabra y una serie de palabras del español escrita con letras mayúsculas pequeñas para representar, de la forma más fidedigna posible, el concepto de esa seña. Esta transcripción incluye ciertos elementos propios de la LS como: indicaciones en el espacio, miradas, movimientos corporales y gestos, indicaciones de clasificador y acción construida. Algunos de estos rasgos serán presentados a través de imágenes intervenidas con simbología explicitada.

\section{Resultados}

En relación a la estructura discursiva de las narraciones personales parecieran organizarse en cuatro momentos distintos: I) Introducción; 2) Situación previa al conflicto; 3) Situación de conflicto, con varios momentos y 4) Situación de cierre, que en gramática de las historias se llama resolución y coda en Labov y Walesky (I967).

\begin{tabular}{|c|c|c|c|}
\hline ETAPA & $\begin{array}{c}\text { DESCRIPCIÓN / } \\
\text { ESCENARIO }\end{array}$ & GLOSA & TRADUCCIÓN \\
\hline Introducción & $\begin{array}{l}\text { Relato breve del } \\
\text { eje temático de la } \\
\text { narración. } \\
\text { Escenario: } \\
\text { Este apartado no } \\
\text { tiene escenario } \\
\text { específico, } \\
\text { planteándose como } \\
\text { una declaración. }\end{array}$ & $\begin{array}{l}\text { YO - MAS O } \\
\text { MENOS - OCHO } \\
\text { - ANOOS - } \\
\text { DESCUBRIR -YO - } \\
\text { DIFERENTE - POR } \\
\text { QUÉ }\end{array}$ & $\begin{array}{l}\text { "Yo descubrí por qué } \\
\text { era diferente más o } \\
\text { menos a los } 8 \text { años". }\end{array}$ \\
\hline $\begin{array}{l}\text { Situación } \\
\text { previa al } \\
\text { conflicto }\end{array}$ & $\begin{array}{l}\text { Se relatan eventos } \\
\text { generales, en tiempos } \\
\text { pasados vividos por } \\
\text { los narradores dentro } \\
\text { de estos espacios. } \\
\text { Escenario: } \\
\text { Apertura del } \\
\text { primer escenario, } \\
\text { intercalando dos } \\
\text { espacios; ejemplo: } \\
\text { casa y escuela. }\end{array}$ & $\begin{array}{l}\text { DESPUÉS } \\
\text { - TIEMPO - } \\
\text { AVANZAR } \\
\text { - JUEGOS - } \\
\text { DESAPARECER } \\
\text { - AMPLIAR - } \\
\text { CONVERSACIÓN } \\
\text { - ESO - SENTIR } \\
\text { - DIFERENTE - } \\
\text { YO - TRATAR - } \\
\text { COMUNICACIÓN } \\
\text { - COMO (*) - } \\
\text { ENTENDER }\end{array}$ & $\begin{array}{l}\text { "Después, avanzó el } \\
\text { tiempo, desaparecen } \\
\text { los juegos y se } \\
\text { amplían las } \\
\text { conversaciones". }\end{array}$ \\
\hline
\end{tabular}




\begin{tabular}{|c|c|c|c|}
\hline $\begin{array}{l}\text { Situación de } \\
\text { conflicto } \\
\mathrm{I}^{\circ} \text { momento. }\end{array}$ & $\begin{array}{l}\text { Se desarrolla de } \\
\text { manera gradual, } \\
\text { desde aspectos } \\
\text { más generales de } \\
\text { la vivencia hacia la } \\
\text { situación específica o } \\
\text { conflicto nuclear. } \\
\text { Escenario: } \\
\text { Movimiento y acote } \\
\text { de los espacios, por } \\
\text { ejemplo: pasar de la } \\
\text { escuela a las sesiones } \\
\text { de terapia de habla o } \\
\text { clases realizadas en la } \\
\text { escuela. }\end{array}$ & $\begin{array}{l}\text { TAMBIEN-CASA- } \\
\text { NOSOTROS } \\
\text {-CUATRO-SI } \\
\text { - MAMÁ -PAPÀ- } \\
\text { JUNTOS- [CL: } \\
\text { ALREDEDOR - } \\
\text { MESA-SENTADOS] }\end{array}$ & $\begin{array}{l}\text { "En la casa también. } \\
\text { Vivíamos los cuatro, } \\
\text { mi mamá, mi papá y } \\
\text { mis cuatro bermanos. }\end{array}$ \\
\hline $\begin{array}{l}\text { Situación de } \\
\text { conflicto } \\
2^{\circ} \text { momento }\end{array}$ & $\begin{array}{l}\text { Se da la situación } \\
\text { puntual de } \\
\text { descubrimiento. } \\
\text { Escenario: } \\
\text { Cambio de escenario, } \\
\text { restringiéndose } \\
\text { más el espacio en el } \\
\text { cual se desarrolla la } \\
\text { acción, por ejemplo: } \\
\text { pasar de la casa al } \\
\text { comedor. }\end{array}$ & $\begin{array}{l}\text { ALMORZAR - } \\
\text { SIEMPRE-SONAR } \\
\text { - TELËFONO - } \\
\text { PRIMERO - QUIEN } \\
\text { - RESPONDER } \\
\text { - PRIMER- } \\
\text { HERMANO- } \\
\text { MAYOR-YO [CL: } \\
\text { RESPONDER- } \\
\text { TELÉFONO] } \\
\text { - COLGAR) } \\
\text { - INFORMAR - } \\
\text { ELLOS } \\
\text { ALMORZAR- } \\
\text { SONAR } \\
\text { TELÉFONO- } \\
\text { [CL: CUATRO, } \\
\text { ORGANIZADOS } \\
\text { POR- EDAD } \\
\text { - SEGUNDO } \\
\text { HERMANO) } \rightarrow \\
\text { TELÉFONO - [AC: } \\
\text { hablar por teléfono, } \\
\text { y luego informar) } \\
\text { [CL: CUATRO } \\
\text { HERMANOS) } \\
\text { VOLVER- SONAR } \\
\text { TELÉFONO) - } \\
\text { [CL:HERMANO } \\
\text { MENOR) - YO - } \\
\text { (gesto de pregunta) } \\
\text { - DESCUBRIR - }\end{array}$ & $\begin{array}{l}\text { "Siempre que } \\
\text { almorzábamos } \\
\text { sonaba el teléfono y el } \\
\text { primero en responder } \\
\text { era mi bermano } \\
\text { mayor. Luego él nos } \\
\text { informaba". }\end{array}$ \\
\hline
\end{tabular}




\begin{tabular}{|c|c|c|c|}
\hline $\begin{array}{l}\text { Conflicto } \\
\text { nuclear o } \\
\text { nudo }\end{array}$ & $\begin{array}{l}\text { Finalmente es en este } \\
\text { apartado que sucede } \\
\text { el momento crucial } \\
\text { de la narración, } \\
\text { en este caso, el } \\
\text { descubrimiento de ser } \\
\text { una persona sorda. } \\
\text { Acción construida: } \\
\text { Es este espacio } \\
\text { en el cual se } \\
\text { desarrolla el nudo } \\
\text { del descubrimiento } \\
\text { el cual se apoya } \\
\text { generalmente } \\
\text { en uno de los } \\
\text { rasgos discursivos } \\
\text { característicos de la } \\
\text { LS que es la acción } \\
\text { construida o cambio } \\
\text { de rol. }\end{array}$ & $\begin{array}{l}\text { narradora-niña: }[\mathrm{M} \uparrow] \\
\text { MAMA - PORQUE } \\
\text { - HERMANOS - YO } \\
\text { - VEO - MAYOR } \\
\text { - YO - TAMBIÉN } \\
\text { - PUEDO- } \\
\text { RESPONDER- } \\
\text { TELÉFONO. } \\
\text { mamá: [M } \downarrow] \\
\text { PORQUE - TU - } \\
\text { NO - PUEDER - TU } \\
\text { - SORDA - TU - } \\
\text { TELÉFONO - NO } \\
\text { PUEDER } \\
\text { [Mov. Corporal - } \\
\text { hombro]; [G. Cara] } \\
\text { - [M } \uparrow] \\
\text { narradora-niña: YO - } \\
\text { SORDA - NO [Mov. } \\
\text { Cabeza] [G. pregunta } \\
\text { y negación) - YO - } \\
\text { PODER } \\
\text { mamá:[M } \downarrow] \text { NO- } \\
\text { PODER }\end{array}$ & $\begin{array}{l}\text { Narradora-niña: } \\
\text { "mamá ¿por qué mis } \\
\text { bermanos pueden } \\
\text { responder el teléfono? } \\
\text { yo soy mayor. Yo } \\
\text { puedo". } \\
\text { Mamá: porque tú } \\
\text { no puedes, tú eres } \\
\text { sorda. } \\
\text { Narradora-niña: } \\
\text { ¿yo soy sorda? Yo } \\
\text { puedo responder } \\
\text { el teléfono. } \\
\text { Mamá: no, no } \\
\text { puedes. }\end{array}$ \\
\hline $\begin{array}{l}\text { Cierre de la } \\
\text { narración } \\
\text { - coda - } \\
\text { resolución }\end{array}$ & $\begin{array}{l}\text { Al final del relato, } \\
\text { en la construcción } \\
\text { del cierre se utilizan } \\
\text { preferentemente } \\
\text { construcciones } \\
\text { metafóricas, para } \\
\text { explicar el darse } \\
\text { cuenta de la } \\
\text { situación. }\end{array}$ & $\begin{array}{l}\text { DARSE CUENTA } \\
\text { - [MET: AJUSTAR } \\
\text { IDEAS - DARSE } \\
\text { CUENTA] } \\
\text { EMPEZAR - } \\
\text { DESCUBRIR - } \\
\text { SORDO - ESO }\end{array}$ & $\begin{array}{l}\text { "Abí, me di cuenta. } \\
\text { Me di cuenta. Abi, } \\
\text { empecé a descubrir } \\
\text { que era sorda". }\end{array}$ \\
\hline
\end{tabular}

Esta estructura parece repetirse, con algunas diferencias, en los cuatro informantes, llamando la atención los espacios marcados en los cuales se organizan los momentos claves del discurso narrativo, determinando claros escenarios en los que transcurren los hechos. A continuación, en la figura l se presenta la distribución espacial de la ubicación de la familia respecto del teléfono en el ámbito del comedor y en la figura 2, la ubicación de la casa respecto a la escuela a la que asistía el señante. 
Figura I

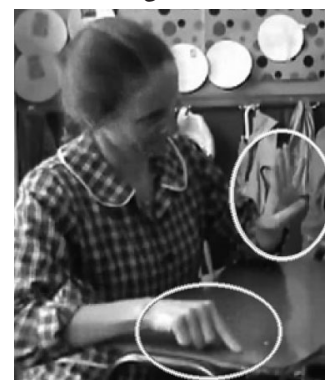

Figura 2

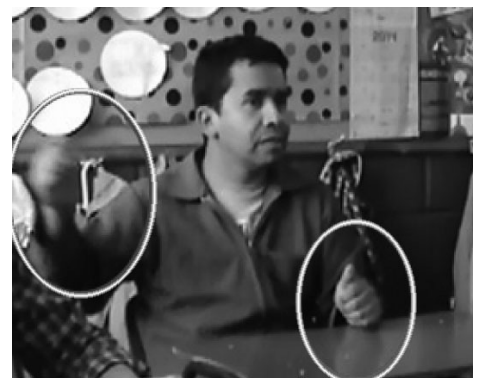

La única acepción la constituyen la introducción y la coda del relato, segmentos en los cuales se resumen los eventos, ya sea para presentarlos o concluirlos. En estos momentos no se da estrictamente una secuencialidad narrativa y, por lo tanto, no se evidencia el espacio en el que transcurren los hechos.

Otro elemento que estas narraciones parecen compartir es la presencia de la acción construida. Este recurso se observa a partir de la representación y desarrollo del diálogo entre los participantes, la interacción entre ellos y los contextos espaciales en los cuales se encuentran. Al igual que en la teoría revisada, la acción construida aquí observada, es una estrategia del discurso que utilizaron todos los participantes a través de gestos con su cara, miradas, movimientos de cabeza y cuerpo. Estos movimientos fueron complementados con señales no manuales, para representar las acciones de un referente, por ejemplo: bablar por teléfono, en la figura 3, conversar desde la perspectiva de la madre o desde la del hermano, con el clasificador del dedo extendido, mostrada en la figura 4.

Figura 3

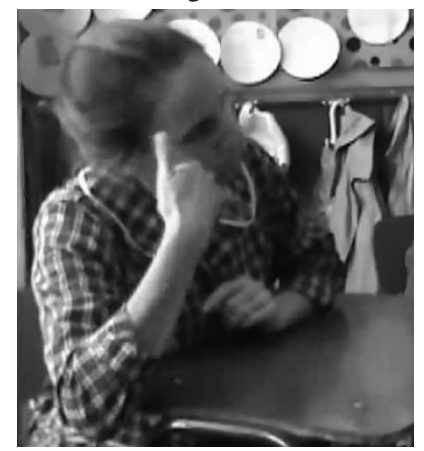


En general, estas construcciones narrativas se utilizaron para mostrar un acceso directo al momento de la situación vivida del nudo, en la cual interactúa el narrador con un interlocutor oyente, generalmente, con un adulto.

Dentro de la acción construida es posible observar la presencia de diálogos, los cuales indican quién es el hablante a través de la direccionalidad de la mirada. Por ejemplo: la mirada del "interlocutor-niño" se dirige hacia arriba cuando dirige su discurso hacia el "interlocutor-adulto" (figura 4); o hacia abajo cuando el rol que se está representando es la del interlocutor adulto que le habla al niño (figura 5), o cuando el hablante tiene la misma altura o rango que el interlocutor (figura 6).

Figura 4

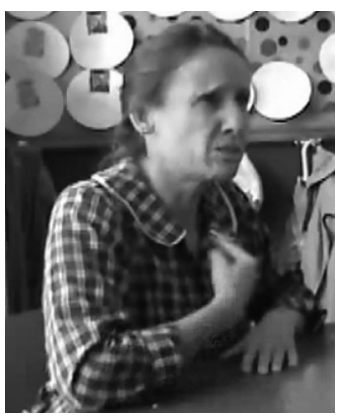

Figura 5

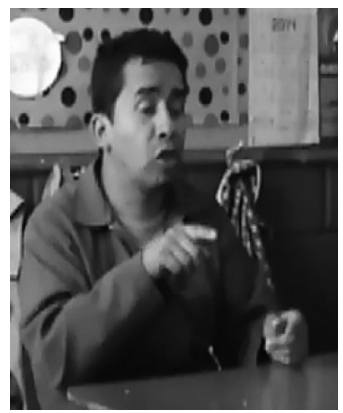

Figura 6

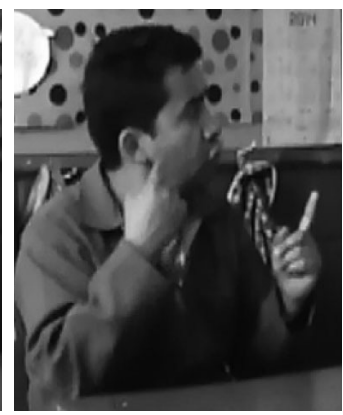

Finalmente, cabe aludir a los clasificadores que aparecen utilizados de manera muy asidua durante el desarrollo de las acciones narrativas, que se observan en el conflicto. Cabe recordar que un clasificador es una seña esquemática que se utiliza para dar un significado, integrando la distribución de un determinado elemento en el espacio. Así, el clasificador no se corresponde exactamente con el formato de la seña, por ejemplo, en la figura 7 se observa el modo de decir comedor en la LSCh, sin embargo, cuando la señante se refiere a que su familia estaba sentada en el comedor, utiliza un clasificador que indica sentados y los organiza con un movimiento en círculo, indicándolos alrededor de una mesa imaginaria. 
Figura 7
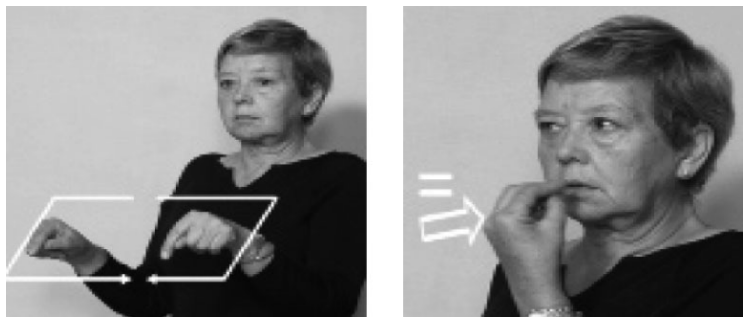

Figura 8

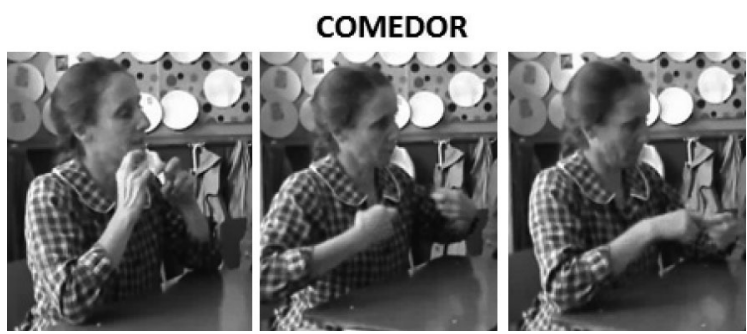

Al final del relato, en la construcción del cierre se utilizan preferentemente construcciones metafóricas, por ejemplo: abí encajan las cosas (figura 9). Estas formas típicas de la LS podrían considerarse como marcadores formulaicos más recurrentes de este tipo de relato.

\section{Figura 9}

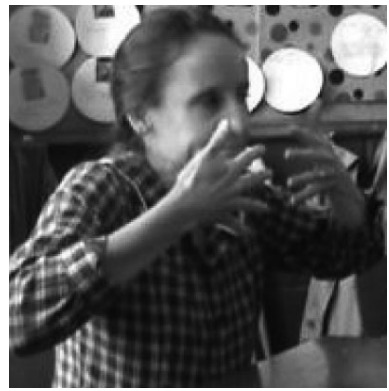

\section{Conclusiones}

El trabajo presentado ha permitido bosquejar algunos de los aspectos centrales de las narraciones LS y, en especial, en LSCh. Tres conceptos importantes parecen desprenderse de este primer acercamiento y permiten entrever algunas sugerencias de elementos 
que deben ser más destacados cuando se utilice la LS en el ámbito educativo bilingüe.

Uno de ellos está relacionado con la estructura en cuatro partes que desarrolla el signante en la narración en lengua de señas, la que parece presentar una forma similar a los episodios de las narraciones orales (Petersen, Gillam \& Gillam, 2008). En este sentido, ambos formatos presentan situación estable-conflicto-resolución, sin embargo, es notoria la necesidad en el usuario de LS de introducir su narración a través de un resumen, que establezca el tema que será desarrollado en el relato. De este modo, el narrador en LS opera de una forma similar a lo que hace un periodista cuando titula una noticia. Es decir, la narración aparece como el desarrollo de algo que ha sido esbozado y activado en la mente del interlocutor.

Dentro de esta estructura hay que destacar el rol fundamental que cumple el espacio no solamente como escenario, sino también como marcador de la continuidad entre los diferentes elementos de la narrativa. Mientras en la oralidad lo preponderante pueden ser las acciones narradas y su organización en un esquema de causa y efecto (Arancibia, Otárola, Figueroa, Manghi, 20I4), en la LS de las personas sordas pareciera cobrar mayor relevancia la ubicación de estas acciones en un determinado lugar representado conjuntamente por ambos interlocutores. Esta importancia central del espacio debe ser potenciada y aprovechada en la elaboración del discurso pedagógico dentro de una propuesta de formación bilingüe, ya que pareciera ser una forma más propia de presentar y acceder a la información en las lenguas visogestuales. En este sentido, se cree que la construcción de escenarios y espacios en ámbito narrativo no solo debe estar presente en el discurso del profesor, sino que debe favorecerse y propiciarse en el discurso de los alumnos.

Otro aspecto importante está relacionado con la necesidad de construir acciones que permitan una comunicación más evidente de los significados y, asimismo, la indicación de los intercambios dialogales a través del uso de la mirada. Estos elementos acercan las LS a los códigos que rigen la mímica y el teatro, elementos que al interior de estos sistemas lingüísticos parecieran configurarse de manera sistemática y que, por lo tanto, han sido estudiados de manera 
muy acuciosa por diversos lingüistas (Oviedo, 1996; Valli \& Lucas, 2000; Emmorey, 2000; Cruz, 2008; Acuña et al., 2012), quienes han destacado su valor para construir la coherencia de los textos. Por ese motivo, se considera que deberían ser destacados y trabajados cuando se produce el desarrollo de las potencialidades comunicativas de los niños sordos en el ámbito de la educación bilingüe y la adquisición/ aprendizaje de la LS.

Finalmente, cabe referir la importancia de los clasificadores en la construcción del discurso narrativo como un componente que complementa y enriquece a la acción construida. Este recurso que es sui géneris en las lenguas de señas debe ser trabajado reflexiva y metacognitivamente en las salas de clases bilingües para concientizar a los estudiantes sobre su potencial comunicativo.

En otras palabras, se entiende que estos recursos propios de la LSCh pueden y deben ser incorporados en el registro pedagógico que ocurre en las aulas bilingües y su potencial semiótico debe ser aprovechado para lograr un desarrollo más profundo e integral de los alumnos sordos que asisten a ellas.

\section{Referencias bibliográficas}

Acuña, X, Adamo, D. \& Cabrera, I. (2009). Diccionario bilingüe lengua de señas cbilena-español. Gobierno de Chile - MINEDUC: Santiago.

Acuña, X., Adamo, D., Cabrera I. \& Lissi, M. (20I2). Estudio descriptivo del desarrollo de la competencia narrativa en LS Chilena. Revista Onomazein, 26, 193-219.

Adamo, D. (1993) Estudio descriptivo de los parámetros básicos de la estructura sub lexical de la LS cbilena. (Tesis de Magíster en Letras con mención en Lingüística). Pontificia Universidad Católica de Chile, Santiago.

Adamo, D., Acuña, X., Cabrera, I. \& Cárdenas, A. (1997). ¿Por qué una educación bilingüe bicultural para las personas sordas? Revista Universidad Metropolitana de Ciencias de la Educación, 3, 43-52.

Adamo, D., Cabrera, I., Lattapiat, P. \& Acuña, X. (1999) Verbo de concordancia en la LS Chilena. Revista Onomazein (4), 335-344.

Adamo, D., Cabrera, I. \& Acuña, X. (2005). La lengua de señas chilena, la cultura y la educación de las personas sordas en Chile. Perspectivas educacionales, 5, 27-33. 
Adamo, D., Cabrera, I. \& Acuña, X. (2008). La educación bilingüe para las personas sordas en Chile. Ponencia presentada en el Encuentro Internacional de Investigación en Lingüística de las LS, Bogotá, Colombia.

Arancibia, M., Otárola, F., Figueroa, A. \& Manghi, D. (2014). Perfiles narrativos en tres poblaciones distintas: Discapacidad Intelectual, Trastorno Específico del Lenguaje y Desarrollo Típico. Ponencia presentada en II Congreso Internacional Educación sin fronteras: Inclusión para el aprendizaje, Temuco, Chile.

Becerra, C. (2008). Metáforas en lengua de señas chilena. Psykbe (Santiago), I7(I), 4I-57.

Cabrera, I. (1993). Descripción de la referencia pronominal en la lengua de señas de las personas sordas de Santiago. (Tesis de Magíster en Letras con mención en Lingüística). Pontificia Universidad Católica de Chile, Santiago.

Crespo, N. (1993). Diccionario de lengua de señas, Córdoba. Córdoba: Grupo de Investigación de Lenguaje de Señas Córdoba.

Cruz, M. (2008). Gramática de la lengua de señas mexicana. (Tesis doctoral). Centro de Estudios Lingüísticos y Literarios, D.F., México.

Earis, H. \& Cormier, K. (2013). Point of view in british sign language and spoken english narrative discourse: The example of "The Tortoise and the Hare”. Language and Cognition, 4 (5), 313-343.

Emmorey, K. (2000). The Confluence of space and language in signed languages. En C. Valli y C. Lucas, (Eds.), Linguistics of American Sign Language: an introduction. (pp. 318-346). Washington, D.C.: Gallaudet University Press.

Emmorey, K., Korpics, F. \& Petronio, K. (2008). The use of visual feedback during signing: Evidence from signers with impaired vision. Journal of Deaf Studies and Deaf Education, I4 (I), 99-I04.

Herrera, V. (2003). Bilingüismo y lectura en la educación de las personas sordas: una propuesta teórica. Revista El Cisne, I59, 2-8.

Herrera, V. (2007). Códigos de lectura en sordos: La dactilología y otras estrategias visuales y kinestésicas. Revista Latinoamericana de Psicología, 2 (39), 269-286.

Herrera, V. (2009). Procesos cognitivos implicados en la lectura de los sordos. Estudios Pedagógicos, 25 (I), 79-92.

Herrera, V. (2010). Estudio de la población sorda en Chile: Evolución histórica y perspectivas lingüísticas, educativas y sociales. Revista Latinoamericana de Educación Inclusiva, I (4), 2 I I-226. 
Ibáñez, A., Becerra, C., López, V., Sirlopú, D. \& Cornejo, C. (2005). Iconicidad y metáfora en el lenguaje chileno de signos (LENSE): Un análisis cualitativo. Revista Electrónica de Investigación y Evaluación Educativa, II (I), $27-45$.

Labov, W. \& Waletzky, J. (1967). Narrative analysis oral versions of personal experience. En J. Helm (Ed.), Essays in the verbal and visual arts (pp. I2-44). Seatle: University of Washington Press.

Liddell, S. \& Metzger, M. (I998). Gesture in sign language discourse. Journal of Pragmatics, 30, 657-697.

Lissi, M.R., Svartholm, K. \& Gonzales, M. (2012). El enfoque bilingüe en la educación de sordos: Sus implicancias para la enseñanza y aprendizaje de la lengua escrita. Estudios Pedagógicos, 2 (38), 299-320.

Lucas, C. \& Valli, C. (1992). Language contact in the American deaf community. Washington: Gallaudet University.

Massone, M. I. (1996). Consideraciones semióticas y discursivas de la lengua de señas Argentina. Ponencia presentada en III Congreso de Bilingüismo. Mérida, Venezuela.

Mayan M. (200I). Una introducción a los métodos cualitativos. Módulo de entrenamiento para estudiantes y profesionales. Alberta: International Institute for Qualitative Methodology.

Metzger, M. (1995). Constructed dialogue and constructed action in american sign language. En C. Lucas (Ed.), Sociolinguistics in Deaf Communities, (pp.255-27I). Gallaudet University Press, Washington, DC.

Minami, M. (2002). Culture-specific language styles. Frankfurt: Multilingual Matters.

Miranda, M. (1997). Evaluación de la comprensión lectora en alumnos sordos. (Tesis de Magíster). Universidad Metropolitana de Ciencias de la Educación, Santiago.

Morgan, G. (2005). Transcription of child sign language: A focus on narrative. Sign Language and Linguistics, 8 (I-2), II9 - I30.

Mulrooney, K. J. (2009). Extraordinary from the ordinary: Personal experience narratives in american sign language. Washington, DC: Gallaudet University Press.

Oviedo, A. (1996). Contando cuentos en lengua de señas venezolana. Mérida, Venezuela: Universidad de los Andes.

Owens, R. (1995). Language disorders: a functional approacb to assessment and intervention. Boston: Allyn \& Bacon. 
Petersen, D., Gillam, S. \& Gillam R. (2008). Emerging procedures in narrative assessment the index of narrative complexity. Top Lang Disorders. 28, 2, II5-I30.

Pilleux, M. (1992). Diccionario del lenguaje de señas de Concepción. Ediciones Universidad Austral. Valdivia.

Schembri, A. (2003). Rethinking "classifiers" in signed languages. En K. Emmorey (Ed), Perspective on classifier constructions in sign languages (pp. 3-34). New York: Psychology Press.

Schembri, A., Fenlon, J., Johnston, T. \& Cormier, K. (2013). Documentary and corpus approaches to sign language research. En E. Orfanidou, B. Woll \& G. Morgan (Eds.), The Blackwell guide to research metbods in sign language studies. Oxford: Blackwell.

Skliar, C. (1998) Bilingüismo y biculturalismo: Un análisis sobre las narrativas tradicionales en la educación de los sordos. Revista Brasileira de Educação, $8,44-57$.

Slobin, D., Hoiting, N., Kuntz, M., Lindert, R., Weinberg, A., Pyers, J., Anthony, M., Biederman, Y. \& Thumann, H. (2003). A cognitive, functional perspective on the acquisition of 'classifiers'. En K. Emmorey, (Ed.), Perspectives on classifier constructions in sign languages (pp. 27I-298). Mahwah, NJ: Lawrence Erlbaum.

Stokoe, W. (1993 [1960]). Sign language structure. An outline of the visual communication systems of the American deaf. Silver Spring: Linstok Press.

Stromqvist, S \& Verhoeven, L. (2004) Typological and contextual perspectives on narrative development. En S. Strömqvist, \& L.T. Verhoeven (Eds.). Relating Events in Narrative (2), Typological and Contextual Perspectives. New Jersey: Lawrence Erlbaum.

Thompson, R., Emmorey, K., \& Kluender, R. (2006). The relationship between eye gaze and verb agreement in american sign language: An eye-tracking study. Natural Language E Linguistic Theory, 24(2), 57 I-604.

Valli, C. \& Lucas, C. (2000). Linguistics of american sign language: an introduction. Washington, D.C.: Gallaudet University Press.

Wilcox, S., Scheibman, J., Wood, D., Cokely, D. \& Stokoe, W. (I994). Multimedia dictionary of american sign language. En Proceedings of the first annual ACM conference on Assistive technologies (pp. 9-I6). ACM.

Woodward, J. (1978). Historical bases of american sign language. En S. Patricia (Ed.). Understanding language through sign language research, (pp. 2I I-2I8). New York: Academic Press. 